\title{
Wie der Männermangel nach dem 2. Weltkrieg uneheliche Geburten beeinflusste
}

\section{Carina Steckenleiter}

\section{Relevanz}

Bewaffnete internationale, nationale, und regionale Konflikte führen zu hohen Opferzahlen in den Wehrmächten und in der Bevölkerung. Dabei sind Frauen und Männer unterschiedlich stark betroffen. So schreibt z. B. die NZZ (Aug. 2014), dass ca. 85 \% der Todesopfer des Syrienkriegs Männer sind. Am Beispiel des 2. Weltkriegs zeigt die Studie von Bethman und Kvasnika, dass der hohe Verlust von Männern langfristige Auswirkungen auf die Fertilität in den betroffenen Regionen hat.

Beatrix Eugster

\section{Quelle}

Der nachfolgende Text ist eine Zusammenfassung von: Bethmann, D., \& Kvasnicka, M. (2013). World War II, Missing Men and Out of Wedlock Childbearing. Economic Journal, 123(567), 162-194.

Was passiert in einer Gesellschaft, wenn es viel weniger Männer als Frauen gibt? Welche Auswirkungen hat ein Männermangel auf Fertilität und insbesondere auf uneheliche Geburten? Dieser Frage sind Dirk Bethmann von der Korea University und Michael Kvasnicka von der Universität Magdeburg anhand Daten aus der Vor- und Nachkriegszeit in Deutschland nachgegangen.

C. Steckenleiter $(\bowtie)$

Universität St. Gallen, St. Gallen, Schweiz

E-Mail: carina.steckenleiter@unisg.ch 
Die ungeheuren Verluste im 2. Weltkrieg hatten in Deutschland einen starken Männermangel in der Nachkriegszeit zur Folge. So waren von den Geburtsjahrgängen 1910-1927, die komplett zur Wehrmacht eingezogenen worden waren, im Durchschnitt $30 \%$ der Männer gestorben. Daraus resultierte ein starker Frauenüberschuss im heirats- und fortpflanzungsfähigen Alter in Deutschland. Die Studie von Bethmann und Kvasnicka analysiert in diesem Zusammenhang, wie sich dieser Frauenüberschuss auf das Geburtsverhalten und insbesondere auf den Anteil an unehelichen Geburten in der unmittelbaren Nachkriegszeit von 1946-1948 auswirkte. Hierfür nutzen die Autoren Daten bayerischer Landkreise aus der Vor- und Nachkriegszeit. Die Bevölkerung des Bundeslands Bayern entsprach damals in etwa der Hälfte der Gesamtbevölkerung der amerikanischen Besatzungszone.

Der durchschnittliche Anteil an unehelichen Geburten hat sich von 1939 zu 1946 in den bayerischen Landkreisen von 9,2 \% auf 20,8 \% erhöht.

Wenn man die bayerischen Statistiken für die Jahre 1939 und 1946 in Bezug auf aussereheliche Geburten sowie Anzahl von Männern zu Frauen in den Landkreisen vergleicht, fallen markante Unterschiede auf (vgl. Abb. 1). So betrug der Anteil der unehelichen Geburten an allen Geburten 1939 im Durchschnitt 9,2 \%

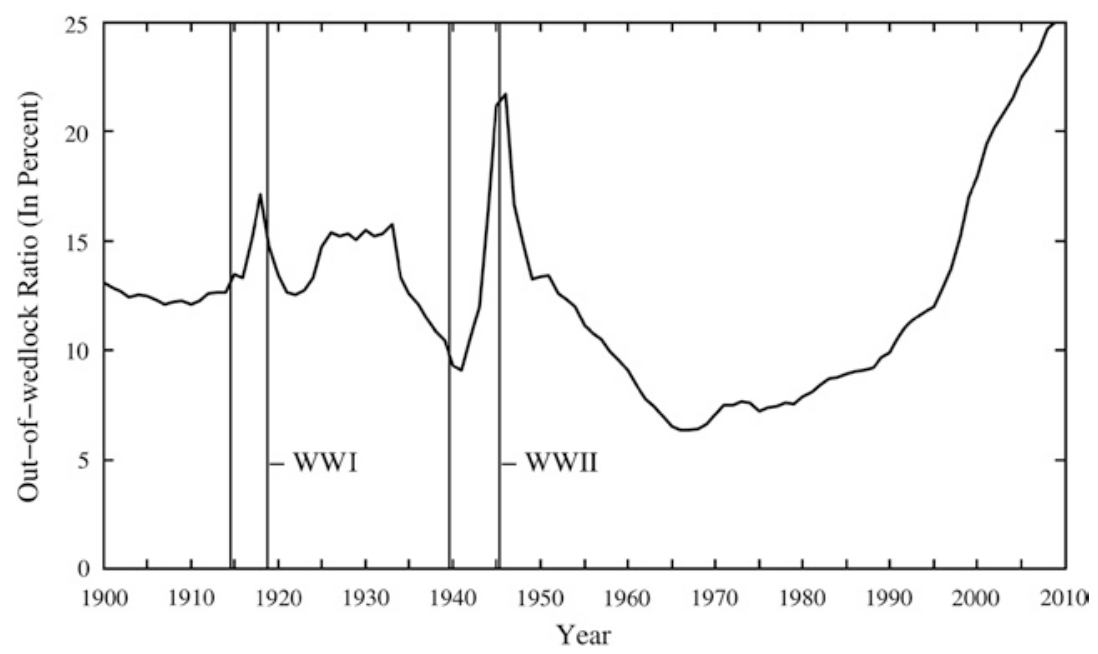

Abb. 1 Anteil an ausserehelichen Geburten per 100 Frauen in Bayern von 1900 bis 2010. (Quelle: Bethmann und Kvasnicka 2013, 172) 
in den bayerischen Landkreisen. 1946 war der durchschnittliche Anteil von ausserehelichen Geburten auf 20,8 \% angestiegen. Somit war 1946 im Schnitt jede fünfte Geburt ausserehelich und der durchschnittliche Anteil von ausserehelichen Geburten hatte sich von vor zu nach dem Krieg um mehr als $125 \%$ erhöht.

Da für die Fragestellung die Relation von Männern zu Frauen im heiratsfähigen Alter relevant ist, stellen die Autoren die Anzahl an Männern im Alter von 20 bis 50 der Anzahl an Frauen im Alter von 14 bis 40 Jahren gegenüber. In dieser Altersgruppe kamen 1939 im Schnitt 95 Männer auf 100 Frauen. Nach dem Krieg war dieses Verhältnis stark gesunken. So kamen im Zensus 1946 im Durchschnitt nur noch 76 Männer auf 100 Frauen. Jedoch war der Ausmass des durch den Krieg entstandenen Männermangels nicht über alle Landkreise hinweg gleichmässig. So gab es Landkreise, in denen sich das Verhältnis von Männern zu Frauen von 1939 zu 1946 besonders stark verschlechtert hatte. In anderen Landkreisen hingegen hatte sich das Geschlechterverhältnis nicht allzu stark verschlechtert oder war nahezu gleichgeblieben. Bethmann und Kvasnicka argumentieren, dass diese durch den Krieg hervorgerufen Veränderungen im Geschlechterverhältnis zufällig waren. So können sie stark und weniger stark betroffene Landkreise vergleichen. Sie untersuchen, ob sich der Anteil an ausserehelichen Geburten signifikant stärker in jenen Landkreisen erhöhte, in denen sich das Geschlechterverhältnis besonders stark verschlechtert hatte.

Da der 2. Weltkrieg Zerstörung und soziale Umwälzungen von nie da gewesenem Ausmass mit sich brachte, beziehen die Autoren weitere Faktoren in ihre Analyse mit ein. Diese sollen für Effekte des Krieges auf Grösse und Zusammensetzung der Bevölkerung, Lebensstandard sowie sozialen Zusammenhalt kontrollieren. So berücksichtigen sie die Bevölkerungsdichte, den Zerstörungsgrad des Hausbestands sowie den Anteil an Heimatvertriebenen in den Landkreisen in ihrer Analyse. Darüber hinaus verwenden sie als Mass für vorherrschende soziale Normen den Anteil an Katholiken in der Bevölkerung.

Die Reduktion des Geschlechterverhältnisses um $1 \%$ führt zu einem Anstieg des Anteils ausserehelicher Geburten von 1,2\% in 1946.

In ihrer Analyse kommen die Autoren zu dem Ergebnis, dass eine Reduktion des Geschlechterverhältnisses um $1 \%$ zu einem Anstieg am Anteil ausserehelicher Geburten von 1,2 \% im Jahr 1946 führt, wenn man die oben genannten zusätzlichen Einflussfaktoren berücksichtigt. Dieser Effekt ist auch für die Jahre 1947 und 1948 noch messbar, fällt jedoch in seiner Grösse ab. In einem zweiten Schritt analysiert die Studie, wie Erwartungen über eine mögliche Verbesserung des Männermangels in naher Zukunft uneheliche Geburten beeinflussen. Um Klarheit 
über das Ausmass an militärischen Verlusten, vermissten Soldaten sowie Kriegsgefangenen zu erhalten, wurden 1946 und 1947 aufwändige Zählungen durchgeführt. Während die Wahrscheinlichkeit, dass vermisste Soldaten noch am Leben waren, sehr gering war, war es bei Kriegsgefangenen sehr viel wahrscheinlicher, dass diese in nicht allzu ferner Zukunft aus der Kriegsgefangenschaft nach Hause zurückkehren würden.

Wenn somit der Frauenüberschuss in einem Landkreis vor allem darauf zurückzuführen war, dass viele Männer gestorben oder vermisst waren, standen die Chancen auf eine zeitnahe Verbesserung des Männermangels wesentlich schlechter als in einem Landkreis, dessen Männermangel durch eine hohe Anzahl an Männern in Kriegsgefangenschaft verursacht war. In ihren Untersuchungen kommen die Wissenschaftler zum Ergebnis, dass in Landkreisen, in denen Kriegsgefangene einen höheren Anteil der Männer im Jahr 1939 ausmachten, sich der Anteil an unehelichen Geburten weniger stark erhöhte. Somit kommt die Studie zum Schluss, dass nicht nur der unmittelbar nach dem Krieg vorherrschende Männermangel, sondern auch Erwartungen, wie sich dieser in naher Zukunft entwickeln würde, das Fertilitätsverhalten beeinflusst haben.

Open Access Dieses Kapitel wird unter der Creative Commons Namensnennung 4.0 International Lizenz (http://creativecommons.org/licenses/by/4.0/deed.de) veröffentlicht, welche die Nutzung, Vervielfältigung, Bearbeitung, Verbreitung und Wiedergabe in jeglichem Medium und Format erlaubt, sofern Sie den/die ursprünglichen Autor(en) und die Quelle ordnungsgemäß nennen, einen Link zur Creative Commons Lizenz beifügen und angeben, ob Änderungen vorgenommen wurden.

Die in diesem Kapitel enthaltenen Bilder und sonstiges Drittmaterial unterliegen ebenfalls der genannten Creative Commons Lizenz, sofern sich aus der Abbildungslegende nichts anderes ergibt. Sofern das betreffende Material nicht unter der genannten Creative Commons Lizenz steht und die betreffende Handlung nicht nach gesetzlichen Vorschriften erlaubt ist, ist für die oben aufgeführten Weiterverwendungen des Materials die Einwilligung des jeweiligen Rechteinhabers einzuholen.

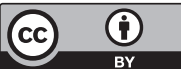

